\title{
Les verbes de déplacement comme contenu d'enseignement du lexique à l'école primaire : modélisation sémantique et analyse de productions d'élèves
}

\author{
Aurnague, Michel $^{1}$, \& Garcia-Debanc, Claudine ${ }^{1,2}$ \\ 'CLLE-ERSS, Université de Toulouse, CNRS, UT2J, France \\ 'SFR AEF ESPE de l'Académie de Toulouse, France \\ michel.aurnague@univ-tlse2.fr \\ claudine.garcia-debanc@univ-tlse2.fr
}

Résumé. La présente contribution se propose de montrer l'intérêt de la référence à une modélisation sémantique pour organiser de façon méthodique un enseignement $\mathrm{du}$ lexique à l'école primaire. Le contenu retenu est celui des verbes de déplacement, fréquemment utilisés dans des productions orales ou écrites narratives, fictionnelles comme scientifiques. Les données analysées proviennent d'une recherche collaborative associant enseignants-chercheurs et enseignants d'école primaire, Didacdep (« Didactique de la description du déplacement »). La référence est constituée par une typologie des verbes de déplacement fondée sur les notions de changement d'emplacement et de changement de relation locative élémentaire. Un ensemble d'activités a été conçu collectivement et mis en œuvre dans six classes d'école primaire : les élèves ont eu successivement à produire des phrases incluant des prédicats de déplacement, à rédiger un texte narratif, à procéder à une classification de verbes partageant certains traits sémantiques identifiés dans la modélisation linguistique (et ce, à partir de deux listes distinctes de prédicats de déplacement) ainsi qu'à éliciter, dans le cadre d'une tâche onomasiologique de production de phrases, des verbes répondant à des propriétés de sens spécifiques (activité ritualisée répétée avec les mêmes consignes). Une tâche d'évaluation finale, sous la forme d'une production écrite narrative, a permis de mesurer les éventuels écarts avec la tâche narrative de début de séquence.

\begin{abstract}
The present contribution aims at showing the interest to refer to a semantic modelling in order to organize, in a methodical way, vocabulary teaching in primary school. Motion verbs have been chosen as field of study because they often appear in oral and written narrative productions, be they fictional or scientific. The data analysed stem from a collaborative research gathering teachers-cum-researchers and primary school teachers, Didacdep ("Teaching of motion description"). The reference consists in a typology of motion verbs grounded on the notions of change of placement and change of basic locative relation. A set of activities has been collectively devised and implemented in six primary school classrooms: students were successively asked, to produce sentences including motion predicates, to write a narrative text, to set up a classification of verbs sharing some meaning features highlighted in the linguistic modelling (the classifications were based on two distinct lists of motion predicates), and to elicite verbs matching specific semantic properties through an onomasiological task of sentence production (ritualized activity repeated with the same instructions). A final assessment task consisting in the production of written narratives allowed us to measure the possible differences with respect to the narrative task at the beginning of the teaching sequence.
\end{abstract}




\title{
1 Une recherche sur l'enseignement du lexique en didactique du français langue première ${ }^{1}$
}

\begin{abstract}
Alors que la question de l'acquisition lexicale et de la mémorisation du vocabulaire est importante en didactique des langues (Van der Linden, 2006), en didactique du français langue première, les recherches portant sur le lexique « occupent une place paradoxale », comme le souligne E. Nonnon dans sa note de synthèse sur les travaux des vingt dernières années, « en ce qu'elle est à la fois marginale (...) et affirmée d'une grande importance, tant par les enseignants que par les injonctions institutionnelles (...), les formateurs et les chercheurs » (Nonnon, 2012 : 33). Grossmann (2011) conclut son «état des lieux » des recherches portant sur « la didactique du lexique » en appelant de ses vœux une meilleure prise en compte de l'enjeu curriculaire (Grossmann, 2011: 180). En effet, au vu de l'hétérogénéité des compétences lexicales des élèves dès l'entrée à l'école, il est crucial d'organiser un enseignement du lexique efficace, en vue de réduire les inégalités sociales. Or, les contenus et les modalités de ces enseignements ne sont pas définis de façon précise dans les programmes français actuels et sont investigués de façon insuffisante dans les recherches en didactique du lexique, avec une prise en compte inégale des différents domaines de l'étude du lexique : morphologie, étymologie, relations sémantiques, lexique-grammaire, lexicographie.
\end{abstract}

La présente étude porte sur un réseau sémantique, celui des verbes de déplacement. Le choix de ce contenu a été doublement motivé, d'une part d'un point de vue praxéologique, et d'autre part d'un point de vue scientifique. En effet, les élèves d'école primaire et de début de collège ont fréquemment l'occasion de décrire des déplacements de personnages ou d'animaux aussi bien dans des récits fictionnels (Masseron, 2001) que dans des textes descriptifs ou explicatifs en biologie (Laparra, 2005). La disponibilité d'un modèle linguistique pour décrire ce réseau sémantique (Aurnague, 2008, 2011) a paru de nature à faciliter la conception d'une ingénierie didactique, en tant qu'ensemble cohérent d'activités à mettre en œuvre dans des classes (Artigue, 1990). Les données analysées dans cette contribution ont été collectées dans le cadre d'une recherche collaborative associant des enseignants d'école primaire et des formateurs d'enseignants, le projet Didacdép («Didactique de la description du déplacement ») mené dans six classes françaises de cycle 3 (élèves de 8 à 11 ans). Les échanges dans ce groupe de travail ont permis de concevoir un ensemble d'activités et d'analyser leur mise en œuvre en s'attachant notamment aux productions des élèves et aux aides lexicales pour la production écrite.

Les activités ont été conçues en référence à un certain nombre de principes guidant la mise en place d'un enseignement méthodique du lexique, qui ont été exposés dans des publications antérieures (GarciaDebanc \& Chourau, 2010). Tout d'abord sont proposées des tâches de production orale ou écrite permettant aux élèves de mobiliser le vocabulaire déjà disponible pour eux, de manière à cerner les besoins mais aussi à favoriser les liens entre vocabulaire nouvellement acquis et vocabulaire actif avant la séquence d'enseignement. Une tâche d'évaluation, en fin de séquence, permettra d'identifier le vocabulaire engrangé par chacun des élèves. Les différentes activités d'enseignement s'inscrivent dans 
une dynamique faisant alterner (a) des apprentissages en contexte, avec l'appui d'œuvres de littérature de jeunesse contenant un nombre important de verbes de déplacement, ici un album de F. Place, Le Roi des trois Orients et un album de C. Van Ahlsburg, L'épave du Zéphyr, et (b) des séances d'étude de la langue, au cours desquelles sont explicitées les significations, les constructions syntaxiques et les conditions d'emploi de verbes de sens proches, avec l'appui de différents dictionnaires. L'explicitation de la signification des verbes est réalisée à la fois en production écrite individuelle dans des tâches d'emploi au sein de phrases, et à travers des activités de classification qui permettent de regrouper des verbes partageant un ou plusieurs traits sémantiques. Ces activités de classification mettent en jeu successivement des tâches réalisées individuellement, des échanges oraux en petits groupes donnant lieu à la réalisation d'affiches ainsi que des moments de mises en commun et de discussions à partir des affiches produites par les différents groupes. Tout au long de la séquence d'enseignement, qui dure plusieurs semaines, sont mises en place de nombreuses activités de réemploi des verbes étudiés, à l'oral et à l'écrit. Ces activités sont complétées par des activités ritualisées, brèves mais fréquentes et se déroulant de manière répétitive, qui doivent permettre la mobilisation des unités lexicales apprises. L'ingénierie didactique construite collaborativement est ensuite mise en œuvre dans chacune des classes, les enseignants y apportant les aménagements qu'ils souhaitent.

Dans la perspective de construction collaborative d'une ingénierie didactique, la référence à une modélisation linguistique est nécessaire et intervient à divers niveaux du dispositif. Elle permet tout d'abord de contrôler les corpus soumis aux observations des élèves, qu'il s'agisse de textes ou de listes de verbes. Elle offre également un cadre théorique pour l'analyse des productions des élèves et l'explication de leurs erreurs, voire l'anticipation de ces erreurs. Elle fournit enfin aux enseignants les éléments-clés autour desquels peuvent être organisées les mises en commun des propositions élaborées par les élèves. La structuration des unités lexicales qui en résulte prend notamment la forme d'affichages constituant des ressources en termes d'aides lexicales pour la production écrite (Garcia-Debanc, 2013).

Dans le cadre de cette contribution, nous nous attacherons principalement à une analyse des productions individuelles des élèves au cours des séances d'étude de la langue pour mettre en évidence les intuitions sémantiques qu'elles manifestent. Il sera, pour l'essentiel, fait état de la classification des verbes par les enfants et d'une activité ritualisée. Les autres tâches du dispositif global, productions écrites narratives de début et de fin de séquence notamment, ne seront pas traitées ici (l'analyse de ces données est en cours, voir Garcia-Debanc \& Aurnague, 2015).

Nous exposerons tout d'abord la modélisation linguistique des verbes et procès de déplacement qui a constitué la référence centrale de notre projet (section 2). Nous présenterons ensuite le protocole de recueil des données (section 3) puis les résultats relatifs aux emplois et aux classifications de verbes de déplacement par des élèves de 8 à 11 ans (section 4). Nous examinerons successivement les verbes de déplacement utilisés par les élèves des trois niveaux scolaires dans des productions de phrases (4.1), les classifications individuelles d'une liste de verbes indiquant le «changement d'emplacement» (4.2) et enfin l'élicitation des verbes de changement d'emplacement dans une activité onomasiologique ritualisée (4.3). Une cinquième section comportera un bilan de nos principaux résultats, l'analyse de quelques extraits d'échanges oraux collectifs permettant aux élèves de confronter leurs propositions de classification, avec l'aide des interventions de l'enseignant, ainsi que les prolongements et perspectives d'analyses complémentaires des données collectées dans le cadre de ce projet.

\section{La modélisation linguistique des verbes et procès de déplacement}

Comme il a déjà été évoqué, les activités proposées aux élèves dans le cadre du projet Didacdép sont basées sur une modélisation sémantique fine des items linguistiques mobilisés et, tout particulièrement, des verbes de déplacement. Les prédicats spatiaux dynamiques auxquels nous nous intéressons sont des verbes intransitifs - transitifs «indirects » inclus - décrivant le déplacement «autonome » d'une entité mobile (seuls quelques verbes transitifs directs apparaissent dans les activités : atteindre, quitter) : aller à, arriver, déraper, descendre, entrer, foncer, se hisser, partir, ramper, se rendre, sortir... Ils se distinguent à la fois syntaxiquement et sémantiquement des prédicats spatiaux dynamiques (généralement transitifs 
« directs ») dénotant un déplacement dont le caractère «causé » ou induit est rendu explicite par l'identification (via le sujet syntaxique) de l'agent à l'origine du procès : amener, apporter, conduire, emporter, traîner, transporter...

Depuis au moins deux décennies, les travaux syntactico-sémantiques sur l'expression du déplacement dans la langue sont parcourus par une opposition récurrente entre les verbes de déplacement autonome véhiculant la manière de se mouvoir et ceux qui indiquent un déplacement au sens strict (ex : Jackendoff, 1990, Levin, 1993, Levin \& Rappaport, 1992, 1995) : foncer, grimper, marcher, zigzaguer vs. arriver, partir, sortir, se rendre. Le recours à cette distinction trouve sans doute son origine dans le contraste fondateur établi par L. Talmy (1985) entre l'expression de la manière du déplacement et celle du trajet ou 'path'. De nombreuses analyses linguistiques ont tenté de saisir l'opposition entre manière et déplacement strict via l'aspect interne ou mode d'action des verbes (ex : Dini \& Di Tomaso, 1999) : alors que la première notion donnerait naissance à des procès atéliques - pouvant se prolonger indéfiniment puisque non pourvus de borne ou transition intrinsèque : foncer, grimper, marcher, zigzaguer -, la seconde se matérialiserait dans des prédicats transitionnels qui mettent, par conséquent, en jeu la télicité - ex : arriver, partir, sortir, se rendre. Le traitement aspectuel de la distinction mentionnée ci-dessus n'est pas sans poser problème (Aurnague, 2012) et nous avons, pour notre part, préféré en rendre compte en recourant à des concepts spatio-temporels qui seront, dans un second temps, mis en relation avec le comportement des prédicats en termes de mode d'action.

Pour autant, la mise au jour de concepts spatio-temporels appropriés n'est pas une tâche aisée, comme le montrent les problèmes que soulève une caractérisation des verbes de déplacement strict en termes de changement de lieu (Laur, 1991, Randall, 2010) ou de déplacement dirigé inhérent (Levin, 1993). Alors que la première notion catégorise comme des «lieux » des entités qui n'en sont pas (et tend à réduire la sémantique des relations spatiales à une relation géométrique d'inclusion dans une région), la seconde recourt à un critère qui n'est non seulement pas exclusif des prédicats de déplacement strict, mais semble vérifié par peu d'entre eux (pour plus détails, voir Aurnague, 2008, 2011). Une définition spatiotemporelle des verbes et procès de déplacement est cependant possible. Elle doit, selon nous, faire appel aux deux notions distinctes que sont le changement d'emplacement et le changement de relation locative élémentaire. Une entité-cible ou entité localisée participe à un changement d'emplacement (également dénommé «translocation» dans d'autres travaux) si sa position dans le cadre de référence terrestre varie au cours du procès considéré : contrairement à s'asseoir qui n'implique pas de déplacement dans le cadre de référence englobant, marcher est bien porteur de cet état de fait. La notion de changement de relation locative élémentaire due à Boons (1987) suppose, quant à elle, que la relation entre une entité-cible et l'entité-site par rapport à laquelle s'opère la localisation soit modifiée durant le procès : les énoncés Max a marché dans la prairie et Max est entré dans la prairie ont ceci de différent que seul le second met en jeu un changement de relation locative (être dans) par rapport au site désigné par le groupe nominal la prairie.

Les deux concepts ainsi mis en évidence dessinent une combinatoire complexe à partir de laquelle quatre catégories de verbes et procès peuvent être définies (voir Tableau 1). Les changements d'emplacement sans changement de relation, évoqués plus haut à propos de marcher, regroupent, en particulier, les verbes de déplacement généralement considérés comme relevant de la manière. A l'opposé, un changement de relation locative élémentaire peut intervenir sans que soit impliqué aucun changement d'emplacement, ce qui se produit, par exemple, pour les prédicats sous-tendus par la relation spatiale de support/contact (être sur; ex : se poser). Les verbes de déplacement strict qui sont au centre de notre modélisation linguistique combinent, comme on peut le constater, un changement de relation et un changement d'emplacement (voir ci-dessous). Enfin, certains verbes et procès ne mobilisent aucune des deux notions utilisées : appartiennent (entre autres) à cette catégorie la plupart des prédicats de changement de posture (ex : s'accroupir, s'asseoir, se recroqueviller) ainsi que les verbes décrivant la façon spécifique dont l'entité-cible « occupe » le site (ex : se blottir, se cacher, s'embourber, se ficher). 
Tableau 1 : Catégories de verbes et procès de mouvement/déplacement

\begin{tabular}{|l|l|l|}
\hline & $\begin{array}{l}\text { Changement } \\
\text { d'emplacement }\end{array}$ & $\begin{array}{l}\text { Pas de changement } \\
\text { d'emplacement }\end{array}$ \\
\hline $\begin{array}{l}\text { Pas de changement } \\
\text { relation }\end{array}$ & $\begin{array}{l}\text { ex : avancer, foncer, } \\
\text { grimper, marcher, nager, } \\
\text { patrouiller, zigzaguer } \\
\text { (cf. liste B) }\end{array}$ & $\begin{array}{l}\text { ex s'accroupir, } \\
\text { recroqueviller } ; \\
\text { se cacher, s'embourber, } \\
\text { se ficher }\end{array}$ \\
\hline Changement de relation & $\begin{array}{l}\text { ex : aller + Prép, arriver, } \\
\text { partir, sortir, se rendre } \\
\text { (cf. liste A) }\end{array}$ & $\begin{array}{l}\text { ex : se poser [toucher, } \\
\text { frôler] s'immerger; } \\
\text { sauter, bondir }\end{array}$ \\
\hline
\end{tabular}

Au-delà des catégories de verbes ou procès que les deux concepts retenus permettent de circonscrire, l'interaction entre changements de relation et changements d'emplacement doit être observée à l'intérieur même de la classe de prédicats qui associe ces concepts, à savoir les verbes de déplacement strict. Le sémantisme de ces verbes (ex : aller + Prép, arriver, partir, sortir, se rendre) fait apparaître deux schémas spatio-temporels principaux (Aurnague, 2008, 2011). Le changement de relation et le changement d'emplacement peuvent, tout d'abord, coïncider temporellement, comme cela se produit pour entrer et sortir, dont la sémantique, basée sur la relation spatiale être dans (changement de relation), se double d'un changement d'emplacement concomitant. Dans d'autres cas, les deux changements sont temporellement disjoints, le changement de relation par rapport au site étant précédé d'un changement d'emplacement (ex: aller à, se rendre). Une «polarité » peut être associée à chaque verbe de déplacement strict, en fonction de la structure du changement de relation sous-jacent. Elle est initiale lorsque la relation est assertée puis niée (l'information «positive » est première : r ... $\neg \mathrm{r}$; ex. : sortir, être-dans $\ldots \triangleright \neg$ être-dans) et finale dans la configuration symétrique (l'information «positive » est seconde : $\neg \mathrm{r} \ldots \triangleright \mathrm{r}$; ex. : entrer, $\neg$ être-dans $\ldots \triangleright$ être-dans). On parle, de la même façon, de polarité médiane - assertion d'une relation précédée et suivie de sa négation : $\neg \mathrm{r} \cdots \triangleright \mathrm{r} \cdots \triangleright \neg \mathrm{r}-$ mais celle-ci concerne un très petit nombre de verbes et locutions verbales du français (ex. : couper par, passer par) et n'intervient pas dans les activités proposées aux élèves.

Pour compléter cette brève analyse des prédicats de déplacement strict et des interactions entre changements de relation et changements d'emplacement, précisons qu'un certain nombre de verbes de simple changement d'emplacement (voir Tableau 1) peuvent, en présence d'un groupe prépositionnel (GP) spatial adéquat, donner lieu à une lecture dans laquelle un changement de relation (final) s'ajoute au changement d'emplacement initialement dénoté par le verbe (ex : Max a couru/rampé/reculé/glissé dans le jardin). Les prédicats de changement d'emplacement concernés présentent des traits sémantiques bien spécifiques - vitesse, opposition à une force, direction/déplacement linéaire orienté, entraînement par une force - qui ont été regroupés au sein de la notion de «tendancialité » (Aurnague, 2008, 2011).

La modélisation linguistique dont nous venons d'esquisser les contours a permis, on le voit, de dégager un nombre important d'éléments de sens intervenant dans l'élaboration des procès de déplacement : cible (entité localisée), site (entité localisatrice), changement d'emplacement, changement de relation locative élémentaire, polarité, vitesse, opposition à une force, direction, entraînement par une force... L'un des objectifs théoriques des activités imaginées dans le cadre du projet Didacdép était précisément de tester le bien-fondé de ces notions et leur utilisation/maniement par les élèves. Sur un plan psycholinguistique, nous faisons l'hypothèse que la mise en place de tâches de natures diverses (rédaction de textes narratifs, production de phrases, classification/tâche sémasiologique, rituel/tâche onomasiologique) devrait s'accompagner d'accès contrastés aux notions sémantiques que véhiculent les verbes et éclairer ainsi l'étude des processus cognitifs associés. 
Deux listes ont été soumises aux enfants pour les activités de classification, l'une constituée de verbes de changement de relation et d'emplacement (déplacement strict; liste A) et l'autre contenant uniquement des verbes de changement d'emplacement (liste B) :

- aller (à), s'en aller, aboutir, s'échapper, accéder, s'enfuir, arriver, entrer, partir, parvenir, pénétrer, se rendre, sortir, venir, atteindre, fuir, quitter (liste A)

- avancer, couler, courir, dégringoler, déraper, descendre, foncer, dévaler, glisser, grimper, ramper, se hisser, reculer, rouler, monter, se traîner, marcher, nager, patiner, skier (liste B).

Dans la suite de ce travail, nous nous concentrerons essentiellement sur la classification des verbes et sur le rituel d'élicitation inclus dans le protocole de recueil de données (cf. section 3), les éléments lexicaux mis en jeu appartenant tous à la liste B développée ci-dessus. Les verbes concernés sont, on l'a dit, des prédicats de changement d'emplacement mais ils se distinguent par un aspect supplémentaire dans la mesure où leur sémantisme intègre, la plupart du temps, un ou plusieurs des traits relevant de la « tendancialité » (voir plus haut; seuls marcher, nager, patiner et skier font exception à la règle). L'une des questions principales auxquelles tentera de répondre l'examen des résultats est de savoir si ces propriétés de sens sont identifiées et maîtrisées par les élèves au cours des activités effectuées.

\section{Protocole de recueil de données}

L'ingénierie didactique développée pour l'enseignement du lexique doit, selon nous, être guidée par un certain nombre de principes que nous avons déjà eu l'occasion de présenter en détail (Garcia-Debanc \& Chourau, 2010). Nous nous contenterons ici d'en rappeler quelques-uns. Il est, tout d'abord, nécessaire d'isoler, parmi les activités proposées aux enfants, des moments identifiés d'étude de la langue pendant lesquels s'articulent travail du lexique en langue et travail en discours, avec l'appui, notamment, des œuvres de littérature de jeunesse. Plus généralement, nous reprenons à notre compte les propositions de Lieury (1997) visant à favoriser une approche «multimodale» des mots qui combine production, réception, oral et écrit. L'appropriation de nouveaux items lexicaux par les élèves sera, en effet, d'autant plus stable que ceux-ci auront été appréhendés selon les dimensions complémentaires que déterminent ces modalités. Il y a, ensuite, un grand intérêt à aborder l'étude de nouveaux mots en s'appuyant sur le fonctionnement de lexèmes déjà connus ainsi qu'en mettant conjointement en lumière les propriétés sémantiques des items et leur comportement syntaxique. Du point de vue plus spécifique du sens, l'organisation des mots en grands domaines notionnels (espace, temps, sentiments...) permet l'exploration de sous-ensembles cohérents d'unités lexicales (Grossmann, Boch \& Cavalla, 2008, Masseron, 2008, Duvignau \& Garcia-Debanc, 2008), à l'intérieur desquels pourront être mis en lumière les grands types de relations qui structurent le lexique - parmi lesquels la synonymie, l'antonymie, l'hypéronymie ou l'hyponymie (Cruse, 1986) - aussi bien que des relations plus rarement mentionnées telle que la «troponymie » (relation entre verbes introduite par Miller \& Fellbaum $\left.(1992)^{2}\right)$. Enfin, on veillera à évaluer les connaissances des élèves avant et après la séquence d'enseignement.

Un protocole en six tâches inspiré des principes précédents a été mis au point et proposé aux enseignants ayant pris part au projet Didacdép. Ce protocole comprenait :

- a) la rédaction individuelle de phrases intégrant des verbes de déplacement ;

- b) la rédaction individuelle d'un texte narratif à partir du dessin d'animation Micro Loup de R. McGuire et G. Solotareff ;

- c) la classification sémantique - avec explicitation des critères de regroupement - d'un ensemble de verbes de changement de relation et d'emplacement (liste A, section 2), activité qui débute par une classification individuelle, suivie d'une classification par groupes présentée sur affiches (après discussion et explicitation des critères) puis d'une mise en commun au sein de la classe ${ }^{3}$;

- d) la classification sémantique d'un ensemble de verbes de changement d'emplacement (liste B, section 2), selon la même procédure ; 
- e) la réalisation d'activités ritualisées et, tout particulièrement, d'une tâche onomasiologique visant à produire ou éliciter des verbes sur la base d'un contenu sémantique donné (rituel « Léon ») ;

- f) la rédaction individuelle d'un texte narratif à partir d'un extrait du dessin d'animation Micro Loup, sans avoir sous les yeux le texte rédigé en tâche b.

Les résultats discutés dans la suite se focalisent, pour l'essentiel, sur la rédaction individuelle de phrases (tâche a ci-dessus), la classification de verbes de changement d'emplacement de la liste B (tâche d) ainsi que la tâche rituelle de nature onomasiologique (tâche e). Les productions ont été recueillies dans deux classes de la banlieue de Toulouse dont les élèves provenaient de milieux sociaux hétérogènes : classe de Sophie (CE2, $3^{\mathrm{e}}$ année primaire) et classe de Lucile (CM1-CM2, $4^{\mathrm{e}}$ et $5^{\mathrm{e}}$ années primaire). Ajoutons que, tout en préservant la cohérence globale de la trame détaillée plus haut, une certaine liberté a été laissée aux enseignants au moment d'implémenter le protocole d'étude des verbes de déplacement.

\section{Résultats}

Comme indiqué ci-dessus, les résultats synthétisés dans cette section portent sur une classe de CE2 et une classe de CM1-CM2, toutes les deux localisées dans la banlieue toulousaine. Après quelques mots sur la production de phrases (section 4.1), nous examinerons les classifications obtenues pour les verbes de changement d'emplacement de la liste B (CE2; section 4.2) puis nous analyserons les élicitations proposées par les élèves (CM1-CM2) dans le cadre du rituel basé sur les propriétés sémantiques que véhiculent ces mêmes verbes (section 4.3).

\subsection{Production de phrases en CE2 et en CM1-CM2}

La consigne proposée aux élèves pour la production de phrases était relativement libre : « Ecris une/des phrase(s) avec un/des verbe(s) de déplacement».

Nous disposons des productions de 8 élèves du CE2 et de 22 élèves du CM1-CM2. Le nombre de verbes de déplacement autonome différents utilisés par élève a été plus élevé en CE2 qu'en CM1-CM2 mais ce décalage est directement imputable au contenu de la consigne qui proposait, dans le premier cas, mais non dans le second, la rédaction de plusieurs phrases (contenant, le cas échéant, plusieurs verbes) : alors que 3 élèves de CE2 ont produit entre 0 et 5 verbes et 5 élèves en ont proposé de 6 à 10 , les 22 élèves de CM1CM2 ont employé de 1 à 5 verbes de déplacement différents.

Pour ce qui est de la nature des verbes élicités, deux différences principales apparaissent entre les classes. On note, tout d'abord, que plus de $68 \%$ des occurrences produites en CE2 - soit 35 sur $51-$ correspondent à des procès de simple changement d'emplacement (cf. section 2, liste B) : accélérer (1), escalader (1), galoper (1), glisser (1), nager (1), naviguer (1), monter (1), planer (1), se promener (1), survoler (1), se frayer un passage (2), voler (5), courir (7), marcher (7) (voir également certaines constructions en faire + Nact/instr ci-dessous). Inversement, les élèves de CM1-CM2 recourent très majoritairement à des verbes de déplacement au sens strict (plus de 66\%, soit 26 occurrences sur 39) qui introduisent, comme on l'a vu, un changement de relation et d'emplacement : s'enfuir (1), plonger (1), rentrer (1), retourner (1), venir (1), s'échapper (3), partir (4), aller + Prép (14). Remarquons, dans cette dernière liste, l'importance spécifique accordée au verbe aller (+ Prép ; ex : aller à) identifié par Viberg (2002) comme étant un « verbe basique » pour l'expression de l'espace dynamique.

L'autre différence observée a trait à la production, par les CE2, de plusieurs constructions associant le verbe support faire à un nom d'activité ou d'instrument (ex : faire de la luge/de l'équitation/des pas chassés/du sport/du vélo), ces occurrences étant, semble-t-il, le fait d'élèves confrontés à des difficultés plus générales. 


\subsection{Classifications individuelles des verbes de changement d'emplacement en CE2 (liste B)}

La classification des verbes de changement d'emplacement de la liste B a suivi, on l'a dit (section 2), plusieurs étapes successives, depuis le travail individuel jusqu'à la mise en commun en passant par des échanges au sein de groupes d'élèves. Nous examinons, dans la suite, les classifications individuelles recueillies en CE2 sans autre contrainte que la mise à disposition des verbes de la liste B (consigne : «Propose un classement des verbes suivants... ») ${ }^{4}$.

21 élèves de CE2 ont pris part à l'activité de classification et créé divers en-têtes ou labels que nous avons recensés puis regroupés sur la base des notions qu'ils visent à identifier ( 1 copie sur les 21 recueillies ne porte la trace d'aucune classification) $)^{5}$. Un premier ensemble d'en-têtes est utilisé pour faire émerger les propriétés qui sous-tendent le concept de «tendancialité » (cf. section 2). Ces propriétés peuvent être saisies à travers un verbe «choisi »- ex : courir, se traîner, descendre, se hisser, dévaler - ou bien faire l'objet d'une identification plus ou moins directe et explicite - ex: rapide, en faisant exprès (intentionnalité associée à l'opposition à une force), négatif (sens d'une direction), en faisant pas exprès (non intentionnalité inhérente à l'entrâ̂nement par une force) - :

- vitesse (rapide) : courir, rapide

- opposition à une force (difficulté «interne») : grimper, se traîner, ce qui va lent, lent, en faisant exprès

- direction, déplacement linéaire orienté (vertical inférieur) : dégringoler, descendre, négatif

- direction, déplacement linéaire orienté (vertical supérieur) : se hisser, monter, en haut d'en bas

- entraînement par une force : dévaler, dégringoler, tomber, en faisant pas exprès, les mots qui veulent dire des catastrophes.

D'autre en-têtes se rapportent à des notions sémantiques ayant été dégagées dans notre modèle (Aurnague, en préparation) mais auxquelles une place plus secondaire a été faite (elles n'ont, en particulier, pas été formalisées). Il s'agit de la «partomotion» ou mouvement relatif des parties d'une entité (cible) au cours de son déplacement ainsi que du médium ou milieu dans lequel l'entité se meut, paramètre qui apparaît, de fait, intimement lié à la partomotion (à propos de ces propriétés, voir également (Stosic, 2009)). Ces notions ont été formulées par les élèves au moyen de verbes - ex : glisser, patiner, couler -, ou bien de noms et groupes prépositionnels dans le cas des médiums/milieux - ex : l'eau, dans la glace et l'eau, sur la terre - :

- partomotion, glisse : glisser, quand on glisse, patiner

- médium/milieu (eau, glace) : couler ${ }^{6}$, l'eau, dans la glace et l'eau

- médium/milieu (terre) : sur la terre

- médium/milieu (non terrestre) : pas sur la terre.

Hormis un regroupement de verbes censé décrire des déplacements réalisés dans un contexte sportif (label les sports), la plupart des en-têtes restants se voient assignés à des classes «incohérentes » ou « résiduelles ». Le recours à certaines de ces classes semble être dû à des verbes isolés (marcher) ou dont la mise en correspondance sémantique avec d'autres unités lexicales n'est pas immédiate. Il en va ainsi, par exemple, de reculer, seul verbe à décrire un déplacement horizontal/frontal (vers l'arrière) alors que plusieurs verbes directionnels verticaux sont présents dans la liste B. Néanmoins, l'émergence de classes au contenu incohérent est, bien plus souvent, le résultat des choix mêmes effectués par les élèves, soit que les en-têtes/labels utilisés n'aient pas de valeur sémantique suffisamment claire (pour eux; ex: déplacement normal), soit qu'ils définissent un spectre trop large pour opérer des distinctions pertinentes entre les procès de changement d'emplacement traités (ex : aller tout droit, tout ce qui se déplace) ${ }^{7}$.

Les éléments de sens pointés par les divers labels recensés ont été inégalement mobilisés, depuis la notion de sport qui n'est à la base que d'un regroupement, jusqu'à celle de partomotion/glisse qui conduit à la constitution de 10 classes (voir ci-dessous). Mis à part les trois propriétés les moins utilisées (sport, terrestre, non-terrestre), on constate que la grande majorité des éléments de sens isolés par les élèves font partie du cadre linguistique ayant alimenté le projet Didacdép (éléments soulignés par un trait plein ou par un pointillé), ce qui tend à consolider, bien sûr, cet arrière-plan théorique : 
sport $(1)<$ terre $($ stre $)$ vs. non-terre $($ stre $)(2)<$ vitesse $(5)<$ eau-glace, opposition à une force $(6)<$

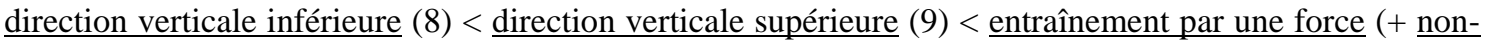
intentionnalité), partomotion/glisse (10).

Le nombre de catégories de verbes structurant les classifications élaborées fait lui aussi apparaître une forte variabilité (cf. Tableau 2) : de 2 classes au plus à 6-9 classes, en passant par un découpage intermédiaire (3-5 classes). Lorsque le nombre de classes créées est mis en regard du degré de cohérence des classifications obtenues (nous considérons incohérentes toutes les productions dont au minimum 50\% des regroupements sont problématiques), il semble, au premier abord, qu'une forte segmentation s'accompagne de davantage d'incongruités (classifications comportant 6 à 9 catégories). Une observation plus fine révèle cependant que le degré de cohérence est beaucoup moins tributaire du nombre de classes/catégories distinguées que de la quantité de verbes traités. Ainsi, les classifications prenant en compte la totalité des 18 verbes listés contiennent-elles beaucoup plus d'incongruités (plus de deux tiers d'entre elles sont «incohérentes » au sens indiqué ci-dessus) que celles établies à partir d'un sousensemble de ces items (un tiers seulement des classifications comprenant de 2 à 17 verbes - avec une moyenne de 10 verbes traités - sont incohérentes), la proportion de classifications complètes incohérentes ne variant que très peu en fonction du niveau de segmentation en classes.

Tableau 2 : Répartition des élèves par nombre de classes créées

\begin{tabular}{|l|c|c|c|}
\hline $\begin{array}{l}\text { Nombre de } \\
\text { classes/catégories }\end{array}$ & $1-2$ & $3-5$ & $6-9$ \\
\hline Nombre d'élèves & 8 & 8 & 4 \\
\hline $\begin{array}{l}\text { Classifications et } \\
\text { nombre de verbes } \\
\text { classés }\end{array}$ & $\begin{array}{c}1 \times 2 ; 2 \times 4 ; 1 \times 9 ; 1 \times \\
13 ; \mathbf{3} \times \mathbf{1 8}\end{array}$ & $\begin{array}{c}1 \times 12 ; 1 \times 13 ; 1 \times 15 ; \\
1 \times 17 ; \mathbf{4} \mathbf{1 8}\end{array}$ \\
\hline $\begin{array}{l}\text { Moyenne des verbes } \\
\text { classés }\end{array}$ & 10,75 & 14,12 & 18 \\
\hline
\end{tabular}

L'une des difficultés auxquelles se trouvent confrontés les élèves dans cette activité de classification est liée au fait que plusieurs des propriétés de sens structurant les changements d'emplacement peuvent soustendre le sémantisme d'un même verbe, cet item étant dès lors susceptible d'apparaître dans divers regroupements ou classes. Cet effet est voulu et il permet aux enfants d'élargir leur connaissance des relations lexicales «classiques» (ex: synonymie, antonymie) en découvrant que des distinctions extrêmement fines peuvent être opérées entre des items lexicaux relevant pourtant d'un même domaine notionnel (voir section 5) - il s'agit, en l'espèce, du déplacement et, plus précisément encore, du changement d'emplacement. La consigne transmise ne faisant aucune référence au caractère disjoint ou non des catégories construites, c'est généralement en cours d'activité que certains élèves demandent des précisions à ce sujet. La plupart des classifications produites dans la classe de CE2 analysée ici semblent, de fait, guidées par une logique de regroupements disjoints et seuls quelques élèves tentent de mettre en place une autre forme d'organisation, en cours de tâche. Ainsi Margot duplique des verbes initialement placés dans une seule classe et Lino incorpore des flèches supposées indiquer qu'un item apparaissant dans une catégorie est également membre d'une ou plusieurs autres classes.

L'examen approfondi des classes construites par les élèves et, tout spécialement, de celles qui présentent une forme d' « hétérogénéité » - pouvant aller jusqu'à l' « incohérence » - nous a permis de montrer que la constitution de ces regroupements suivait parfois des cheminements assez spécifiques ${ }^{8}$. Une catégorie élaborée à partir d'un lexème ou plus $(11 \ldots . .1 n)$ présentant la propriété sémantique A sera, par exemple, élargie à un élément qui ne la possède pas $(\ln +1$; puis éventuellement à plusieurs) en raison de la présence d'un trait $\mathrm{B}$ dans l'un au moins des lexèmes $11 \ldots$ ln ainsi que dans le contenu sémantique de $\ln +1$. En d'autres termes, un regroupement initial de verbes peut croître par focalisations successives sur des propriétés sémantiques plus ou moins distinctes ou éloignées ( $\mathrm{A}$ puis $\mathrm{B}, \mathrm{A}+\mathrm{C}$ puis $\mathrm{B}, \mathrm{A}+\mathrm{B}$ puis $\mathrm{A}$, $A+B$ puis $B$...) sans que cet accroissement relève du hasard. Dans la règle tout juste édictée, le passage d'une propriété $\mathrm{A}$ à une propriété $\mathrm{B}$ nécessite, en particulier, que ces deux éléments de sens soient partie 
intégrante d'un (au moins) des items lexicaux censés partager la première propriété. Un élève du CE2 (Thomas) prend ainsi pour cible initiale un lexème dénotant l'entraînement par une force et la vitesse, à savoir le verbe déraper (en-tête), puis se focalise uniquement sur la seconde propriété pour incorporer les verbes courir et foncer. Recourant à ce même lien entre traits sémantiques (entraînement par une force vitesse) ainsi qu'à la relation entre direction verticale inférieure et entraînement par une force, une autre élève (Camille) forme une classe qui intègre les verbes dégringoler (en-tête) et dévaler (direction verticale inférieure + vitesse + entraînement par une force) aussi bien que déraper (entraînement par une force + vitesse), foncer (vitesse) et descendre (direction verticale inférieure) ${ }^{9}$.

Outre les deux liens déjà cités, d'autres connexions entre propriétés sémantiques semblent avoir conditionné la constitution de certaines classes de verbes par les élèves ${ }^{10}$ : opposition à une force intentionnalité/cible animée ; direction verticale supérieure - opposition à une force ; partomotion/glisse vitesse ; partomotion/glisse - entraînement par une force... Les mécanismes mis en évidence ci-dessus ne manquent pas de rappeler la façon dont s'élaborent certaines catégories conceptuelles et linguistiques dont la «motivation » n'est pas immédiate mais qui répondent néanmoins à des modes de formation spécifiques - ex : «ressemblances de familles»de Wittgenstein (1953) et «catégories radiales » de Lakoff (1987). L'exercice effectué dans le cadre de cette activité de classification était cependant différent, dans la mesure où l'on attendait des enfants qu'ils constituent des catégories plus « classiques » dont tous les membres partagent un ou plusieurs traits communs généralement caractérisés par l'en-tête ou label choisi.

Les productions des élèves de CM1-CM2 ne sont pas exploitables car ceux-ci ont été invités à distribuer les verbes de changement d'emplacement de la liste B en fonction d'un critère prédéfini par l'enseignant («Classe les verbes par rapport au sens de déplacement : horizontal ou vertical »). Par conséquent, elles ne sont pas traitées ici.

\subsection{Elicitation des verbes de changement d'emplacement en CM1-CM2 (rituel « Léon »)}

Une étape importante du protocole mis sur pied consistait en une activité de production de phrases/verbes guidée par la donnée initiale d'une contrainte sémantique. Dans cette optique, plusieurs rituels ont été imaginés par les enseignants, au cours desquels les élèves devaient élaborer des phrases remplissant des critères plus ou moins stricts tels que la nature de l'entité-site impliquée dans le déplacement - ex : désert, forêt, grotte - et, plus rarement, le recours à un verbe spécifique. Les deux classes observées dans le cadre de cette étude ont participé à des rituels de cette nature. Néanmoins, ces activités s'éloignent assez sensiblement de la tâche onomasiologique envisagée en complément de la classification des verbes de changement d'emplacement. Nous nous concentrons donc sur les seuls résultats de la classe de CM1CM2 au sein de laquelle le rituel «Léon » a également été mené sous la forme que nous attendions.

Quatre propriétés sémantiques - constitutives de la notion de tendancialité (voir section 2) - ont été soumises aux élèves pendant la première séance du rituel «Léon » de CM1-CM2, et ce dans l'ordre qui suit : vitesse, direction, difficulté du déplacement (reformulation du concept d'opposition à une force), entraînement par une force. Deux séances planifiées sur deux jours consécutifs ont eu lieu deux semaines plus tard avec, à chaque fois, la possibilité de travailler ou retravailler un trio de propriétés : vitesse, direction, entraînement par une force (séance 2) ; difficulté, déplacement lent, déplacement rapide (séance 3). La consigne utilisée pour chacune des propriétés dont on recherchait l'élicitation par les enfants comprenait une glose, parfois accompagnée d'un exemple, ainsi que la requête/consigne proprement dite. Dans le cas de la vitesse, par exemple, ces deux éléments avaient la forme ci-dessous :

- Léon se déplace(r)/bouge(r) rapidement (glose) [Léon a dégringolé (en bas de l'escalier) (exemple)] - «Produis une phrase avec un (autre) verbe de déplacement indiquant la rapidité » (consigne) ${ }^{11}$.

22 élèves ont participé aux différentes séances du rituel, le nombre de verbes employés par propriété testée pouvant être inférieur à l'effectif total (tous les enfants ne répondent pas) ou, au contraire, le dépasser (certains enfants proposent plus d'une phrase ou intègrent plusieurs verbes dans la même phrase). Avant d'examiner de plus près l'évolution, au fil des séances, du nombre de verbes élicités 
présentant le trait de sens attendu, indiquons que les consignes transmises semblent avoir été comprises par la plupart des élèves. Nous en voulons pour preuve que, lorsqu'ils peinent à produire des verbes répondant au critère sémantique affiché, de nombreux enfants recourent à des éléments cotextuels ou contextuels visant à pallier ce manque. Nous ne ferons pas ici l'analyse détaillée des éléments additionnels ainsi mobilisés et nous contenterons d'en délimiter les principales catégories.

Un premier ensemble d'unités linguistiques est constitué d'adverbes et groupes prépositionnels ayant pour fonction d'introduire plus ou moins directement la propriété sémantique soulignée dans la consigne - alors même que cette propriété est absente du contenu verbal : marcher rapidement (vitesse), nager vers l'île aux palmiers/dans une direction inconnue (direction), se déplacer par force (entraînement par une force)... Dans d'autres productions, c'est un groupe prépositionnel ou un groupe nominal/déterminant qui est intégré à la phrase mais son rôle est assez différent de celui des éléments du groupe précédent, en ce sens qu'il ne dénote pas de façon explicite le paramètre sémantique ciblé mais vise plutôt à installer un contexte situationnel au sein duquel ce paramètre opère : skier la piste noire (vitesse), avancer avec un plâtre à la jambe (difficulté), marcher dans la boue (difficulté) ${ }^{12}$... Enfin, la volonté des élèves de respecter la consigne les conduit parfois à utiliser des constructions syntaxiques potentiellement porteuses de l'élément de sens travaillé dans le rituel. Il s'agit, par exemple, de transmette le sentiment de difficulté en recourant à des phrases dont le verbe principal, au caractère quasi modal, régit une proposition infinitive décrivant un procès spatial dynamique : ne pas arriver à marcher, essayer de se déplacer, galérer à avancer... Dans le même ordre d'idées, l'entraînement par une force aboutit assez souvent à l'usage de verbes et constructions indiquant expressément l' «action sur une entité » (plutôt que le déplacement autonome d'une entité-cible), qu'ils soient à la forme active ou passive : tirer, traîner, faire rouler, être emporté, se faire déplacer ${ }^{13}$...

Ces stratégies supplétives - proches, pour certaines d'entre elles, de la paraphrase - témoignent, comme on l'a dit, du bon niveau de compréhension des consignes par les élèves. Elles révèlent, en même temps, la difficulté qu'il peut y avoir à intégrer ou repérer le trait de sens requis dans le sémantisme verbal luimême. Quelles différences note-t-on, de ce point de vue, entre les diverses propriétés prises en considération et quelles évolutions se dessinent au fil des séances ? C'est ce que nous allons voir maintenant.

Tableau 3 : Proportion d'élèves ayant produit des verbes attendus par séance

\begin{tabular}{|l|c|c|c|c|}
\hline $\begin{array}{l}\text { Propriétés } \\
\text { sémantiques }\end{array}$ & Vitesse & Direction & $\begin{array}{c}\text { Entraînement par } \\
\text { une force }\end{array}$ & Difficulté \\
\hline $\begin{array}{l}\text { Elèves ayant } \\
\text { produit des verbes } \\
\text { attendus } \\
\text { (séance 1) }\end{array}$ & $\begin{array}{c}15 / 22 \\
(68,18 \%)\end{array}$ & $\begin{array}{c}7 / 22 \\
(31,81 \%)\end{array}$ & $\begin{array}{c}(15 \%) \\
(47,61 \%)\end{array}$ \\
\hline $\begin{array}{l}\text { Elèves ayant } \\
\text { produit des verbes } \\
\text { attendus (séances } \\
2 \text { et 3) }\end{array}$ & $\begin{array}{c}19 / 23 \\
(82,60 \%)\end{array}$ & $\begin{array}{c}(40,90 \%) \\
(54,16 \%)\end{array}$ \\
\hline
\end{tabular}

Le Tableau 3 ci-dessus met en regard le traitement par les élèves des quatre propriétés étudiées, au cours de la première séance du rituel d'une part (vitesse, direction, entraînement par une force, difficulté), et lors du renouvellement de ces tâches proposé pendant les séances 2 (vitesse, direction, entraînement par une force) et 3 (difficulté) d'autre part. La vitesse apparaît d'emblée comme une propriété sémantique dont la préhension lexicale - via un verbe de changement d'emplacement - ne pose aucun problème, $68 \%$ des élèves produisant un lexème approprié dès la première séance et plus de $80 \%$ lorsque ce trait est à nouveau travaillé. Ce résultat est conforme à ce qui a pu être observé dans des travaux antérieurs (Garcia-Debanc et al., 2009) où la vitesse semblait être un critère récurrent de classification des prédicats de déplacement par les enfants (voir cependant, plus bas, les différences de nature entre la tâche sémasiologique de classification et l'activité onomasiologique liée au rituel). La production de verbes 
véhiculant le trait sémantique attendu atteint des scores plus modestes dans les trois autres cas. Ainsi, un tiers des élèves $(31,81 \%)$ ayant pris part à la première séance du rituel élicitent des prédicats directionnels, alors que ce critère a été utilisé de façon exclusive dans l'activité de classification, à la demande de l'enseignant. De même, seuls $15 \%$ des élèves produisent des verbes adaptés lorsque ceux-ci sont supposés dénoter l'entraînement par une force. De son côté, l'expression lexicale de la difficulté (au moyen d'un verbe) n'atteint pas tout à fait la barre des $50 \%$ (47,61\%). Malgré des évolutions inégales, la réitération des activités au cours des séances 2 et 3 montre l'utilité du rituel dans la maîtrise des trois propriétés dont le maniement s'avère le plus délicat. Le traitement lexical du concept d'entraînement par une force connaît, en particulier, une progression spectaculaire, des $15 \%$ enregistrés initialement à près de $55 \%$ (soit $261 \%$ de taux d'accroissement). Bien que plus faible, la progression est également sensible pour la propriété de directionnalité $(+28,57 \%)$, avec un tiers des enfants produisant un prédicat adéquat au cours de la première activité rituelle $(31,81 \%)$ et pratiquement $41 \%$ à la reprise de cet exercice. Enfin, les résultats relatifs à la propriété de difficulté changent peu d'une séance à l'autre $(+5 \%$ de taux d'accroissement) mais s'élèvent tout de même à $50 \%$ - soit 1 élève sur 2 produisant un verbe adapté. Sur un plan plus qualitatif, il est intéressant de noter que si les enfants remobilisent spontanément une bonne partie des verbes qui leur ont été soumis dans le cadre de la tâche de classification (liste B remaniée par l'enseignante ; cf. note 4), ils sont aussi capables d'exhiber de nouveaux lexèmes : se dépêcher, sprinter, trotter (vitesse); se diriger, tomber (direction); escalader (difficulté), s'envoler (emploi non intentionnel ; entraînement par une force).

Les différences qui se font jour entre le traitement de la vitesse d'une part, et celui des trois autres propriétés sémantiques impliquées dans le rituel d'autre part, ne sont pas simples à expliquer. Il est possible qu'une production lexicale massive (par un grand nombre d'élèves) de verbes présentant un trait sémantique donné soit la marque d'un accès direct/concret à cette propriété (dans le monde et dans la langue), combiné à une faible complexité - par opposition à un caractère plus abstrait et une complexité accrue. Cette explication reste cependant très générale et un examen approfondi des traits dont le maniement est plus problématique serait nécessaire. Dans le cas de la direction(nalité), par exemple, trois types de facteurs au moins ont été susceptibles de peser sur les résultats. Tout d'abord, peu de verbes de changement d'emplacement dénotant une direction «horizontale » ont été préalablement manipulés par les enfants (reculer dans la tâche de classification (cf. section 4.2) et, le cas échéant, avancer dans l'une des consignes du rituel «Léon»), alors même que de nombreux déplacements verticaux leur étaient proposés : dégringoler, déraper, descendre, dévaler, grimper, se hisser, monter. Deuxièmement, une part importante des prédicats indiquant une direction verticale (supérieure ou inférieure; voir ci-dessus) associaient cette propriété à d'autres traits sémantiques (opposition à une force : grimper, se hisser ; entraînement par une force : dégringoler, déraper, dévaler). Troisièmement, il semble que certains élèves (comme leurs aînés... y compris linguistes !) aient tendance à confondre la notion de direction d'un déplacement et celle de changement de relation locative élémentaire (voir section 2), pensant trouver la trace du premier concept dans le sémantisme de prédicats tels que aller à, arriver, entrer ou partir. Il est pourtant bien établi que la simple directionnalité présente, en termes aspectuels notamment (aspect interne), des propriétés bien différentes de celles mises en jeu par les changements de relation (ex : courir vers et avancer vers sont atéliques alors que courir à et arriver à sont téliques).

Au-delà des comparaisons internes ou intra-tâche, c'est à un rapprochement entre l'activité de classification des verbes de changement d'emplacement (sémasiologique, section 4.2) et l'activité ritualisée (onomasiologique, section 4.3) qu'il faut se livrer. La hiérarchie des concepts utilisés pour la classification (section 4.2) apparaît ainsi sensiblement modifiée lorsqu'on la confronte au Tableau 3. La vitesse qui semble moins prégnante que la difficulté, la direction et l'entraînement par une force lors de la tâche sémasiologique, donne lieu à un maximum de productions lexicales dans les exercices rituels/onomasiologiques. L'inverse se produit pour la difficulté qui, de critère majeur dans les classifications, se convertit en l'élément le moins bien lexicalisé par les enfants invités à lister des verbes prédéfinis (séances 2 et 3 du rituel «Léon ») ${ }^{14}$. L'on pourrait, bien sûr, arguer du fait que, parmi les prédicats de la liste de $\mathrm{B}$, la vitesse est régulièrement combinée à d'autres propriétés (direction verticale inférieure, entraînement par une force : dégringoler, déraper, dévaler...; voir précédemment), celles-ci prenant peut-être l'avantage sur celle-là au moment de classer les lexèmes. Mais ce type de combinaisons 
entre traits est fréquent dans la liste B et pas spécifique à la vitesse. Plus fondamentalement, il est probable que les processus cognitifs mis en jeu dans la tâche sémasiologique de classification soient distincts de ceux qui sous-tendent l'activité onomasiologique de rituel, conduisant dès lors à des modes d'accès différents aux propriétés sémantiques examinées. On peut ainsi, sans trop se risquer, émettre l'hypothèse que l'activité rituelle requiert, de la part des élèves, des opérations sémantiques explicites et complexes qui s'appuient, de ce fait, sur des processus contrôlés, c'est-à-dire moins automatiques (tâches de haut niveau). L'émergence des propriétés de sens par le biais de la classification aurait, comparativement, des bases implicites, prenant dès lors un tour plus « automatisé » (i.e. moins contrôlé ; tâches de bas niveau $)^{15}$.

\section{Bilan et perspectives}

Associé à l'analyse de la classification des verbes de changement de relation et d'emplacement de la liste A (Aurnague \& Garcia-Debanc, à paraître), l'examen des activités élaborées autour des prédicats de changement d'emplacement de la liste $\mathrm{B}$ a permis de montrer que les élèves de cycle 3 de l'école primaire ont accès à des propriétés sémantiques subtiles des verbes de déplacement (parfois associées à des comportements syntaxiques spécifiques: liste A). La combinaison d'une activité sémasiologique de classification et d'une tâche onomasiologique (rituel «Léon») fait, par ailleurs, apparaître que le maniement de ces éléments de sens répond sans doute à des processus et procédés cognitifs différents dans les deux cas. Ces résultats confortent, d'une certaine façon, le modèle linguistique en arrière-plan de l'étude en confirmant, entre autres choses, l'existence et le bien-fondé des propriétés sémantiques à la base de la notion de tendancialité (cf. section 2).

L'examen fin des classifications proposées par les élèves a aussi conduit à la recherche des logiques sousjacentes aux regroupements proposés. Les labels assignés aux catégories peuvent être facilement réinterprétés en référence à la modélisation linguistique. De plus, et au-delà des catégories classiques attendues, il a pu être montré que certains regroupements hétérogènes, voire «incohérents », pouvaient être le résultat d'élargissements successifs d'une catégorie initiale, prédictibles à partir des propriétés lexicales des verbes en jeu.

Hormis la stricte mise à l'épreuve du modèle, l'utilisation d'un cadre d'analyse rigoureux des unités lexicales se révèle, il nous semble, utile pour mesurer les effets en termes d'apprentissages des activités didactiques mises en place. L'activité rituelle, par exemple, conduit les élèves à progressivement mobiliser un plus grand nombre d'unités lexicales verbales porteuses de notions relativement abstraites et complexes (voir, à la section 4.3, l'amélioration, d'une séance à l'autre, de l'appréhension lexicale des propriétés de directionnalité, entraînement par une force et difficulté). Plus généralement, les élèves acquièrent ou activent, au fil des exercices proposés, un éventail assez large de verbes de déplacement dont certains ne sont pas présents dans les deux listes travaillées. Si les effets de cet élargissement lexical requièrent, bien sûr, une évaluation en discours, par l'analyse des productions écrites (voir plus loin), les bénéfices de l'ensemble du protocole mis sur pied ne doivent pas uniquement être mesurés en termes quantitatifs (nombre de verbes maîtrisés et utilisés, dont verbes spécifiques). Les activités proposées modifient, en effet, tout autant l'appréhension qualitative du lexique par les enfants.

Ainsi, lors de la mise en commun des classifications de verbes de changement d'emplacement (liste B) réalisées dans une classe de CM1-CM2 - distincte de celle étudiée précédemment -, une discussion s'engage pour savoir si foncer, courir et avancer sont tous les trois porteurs du trait de vitesse :

171- Professeur des Ecoles : (...) Lesquels il faudrait garder pour être sûr que les...

ceux qui montrent qu'on est sûr d'aller vite?

175- Elève non identifié : Les trois ! foncer, courir et avancer !

176- Groupe d'élèves : Mais non!

178- Hugo : Non pas avancer parce que avancer moi je dis ça je dis rien mais quand

tu avances, tu vas pas vite!

179- Professeur des Ecoles : Quand tu avances tu vas pas forcément vite (...) 
Les controverses entre élèves permettent ainsi de cerner les traits sémantiques de ces différents verbes et de mieux circonscrire ce qui relève du contenu lexical des unités étudiées en le différenciant du cotexte et du contexte situationnel. Les discussions autour de cette question reprennent un peu plus tard et permettent à l'enseignante de montrer aux élèves quels éléments des phrases Je recule à toute vitesse/lentement réfèrent à la vitesse du déplacement. Ces échanges, en petits groupes ou au cours de la mise en commun, suscitent une élaboration progressive d'une vision fine de l'organisation de ces unités (relations lexicales).

Concernant l'organisation du lexique, les activités du protocole permettent de dépasser l'étude des relations habituelles de synonymie, d'antonymie ou d'hypéronymie/hyponymie et d'explorer des phénomènes complexes entre verbes qui relèvent sans doute pour partie de la «troponymie » (voir section 2). Les élèves constatent que des prédicats qui font tous référence au déplacement peuvent être structurés en groupes plus réduits sur la base de critères de sens précis, mais aussi que les éléments de ces sous-groupes sont susceptibles de différer par d'autres aspects et ont, par conséquent, la possibilité d'appartenir à plusieurs ensembles distincts. La séance de travail dont un extrait de la transcription a été donné ci-dessus, commence précisément par un échange enseignant/élèves sur ce qu'est un verbe ou procès de déplacement et se termine par l'observation de ce qui réunit et différencie les éléments d'un même groupe de prédicats. L'enseignante se centre alors sur une classe issue de la mise en commun qui rassemble sous l'en-tête ou label au sol, les verbes ramper, glisser et se traîner :

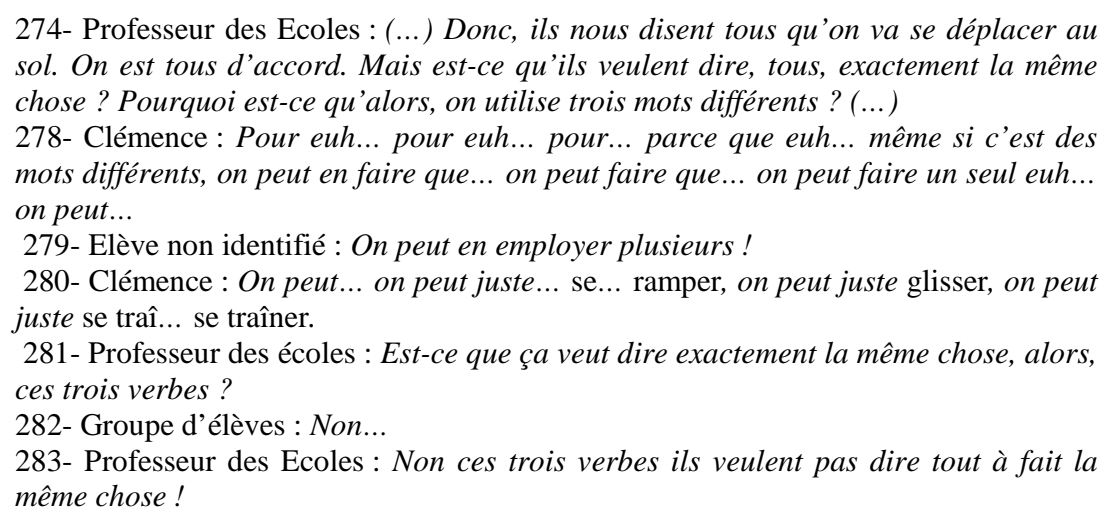

L'enseignante aide ainsi les élèves à prendre conscience à la fois de traits sémantiques communs à un ensemble de verbes et de la spécificité de la signification de chacun d'entre eux.

L'analyse complète du corpus Didacdép suppose, bien sûr, qu'une attention spécifique soit portée aux tâches narratives intégrées au début et à la fin du protocole (voir étapes b et $\mathrm{f}$ à la section 3 ). Ce n'est qu'alors que pourront être appréhendés les possibles effets des activités - de classification notamment sur l'emploi de verbes en production écrite. Cette analyse est en cours (Garcia-Debanc \& Aurnague, 2015). Elle fait apparaître que la diversification des verbes n'est pas aussi importante qu'attendu et qu'elle est mieux repérable dans le cas d'utilisation d'aides lexicales construites à partir des activités de classification. On peut noter toutefois que la diversification des verbes n'est pas une fin en soi, même si elle est souvent privilégiée dans l'évaluation scolaire, mais que le critère décisif est celui de la pertinence des emplois des verbes de déplacement, y compris dans leur construction syntaxique. De ce point de vue, les productions finales sont mieux réussies que les productions initiales.

Un examen approfondi des mises en œuvre de l'ingénierie didactique devra, à plus longue échéance, être également réalisé, afin de déterminer les écarts entre les activités initialement imaginées par les chercheurs et leur implémentation par les enseignants, d'expliciter les critères privilégiés par ces derniers dans les phases de mises en commun et de tenter de cerner les différentes conceptions de l'enseignement du lexique que ces écarts révèlent. 


\section{Références bibliographiques}

Artigue, M. (1990). Ingénierie didactique. Recherches en Didactique des Mathématiques, 9(3), 281-308.

Aurnague, M. (2008). Qu'est-ce qu'un verbe de déplacement ? : critères spatiaux pour une classification des verbes de déplacement intransitifs du français. In : J. Durand, B. Habert \& B. Lacks (éds), Actes du Congrès Mondial de Linguistique Française, CMLF'08. Paris: ILF \& EDP Sciences, 1905-1917 (cd-rom), http://www.linguistiquefrancaise.org/articles/cmlf/pdf/2008/01/cmlf08041.pdf

Aurnague, M. (2011). How motion verbs are spatial: the spatial foundations of intransitive motion verbs in French. Lingvisticae Investigationes, 34:1, 1-34.

Aurnague, M. (2012). De l'espace à l'aspect : les bases ontologiques des procès de déplacement. Corela, HS-12 (Langue, espace, cognition, B. Fagard \& D. Stosic (éds)), http://corela.revues.org/2846

Aurnague, M. (en préparation). Strict autonomous motion in French: elements of semantic modelling.

Aurnague, M. \& Garcia-Debanc, C. (à paraître). Enseignement du lexique à l'école primaire et modélisations linguistiques : exemples d'activités portant sur des verbes de déplacement strict, à paraitre dans Pratiques, 171172.

Boons, J.P. (1987). La notion sémantique de déplacement dans une classification syntaxique des verbes locatifs. Langue Française, 76, 5-40.

Cruse, D.A. (1986). Lexical semantics. Cambridge : Cambridge University Press.

Dini, L. \& Di Tomaso, V. (1999). Linking theory and lexical ambiguity: the case of Italian motion verbs. In : H. Bunt \& R. Muskens (éds), Computing meaning (vol. 1). Dordrecht : Kluwer, 321-337.

Duvignau, K. \& Garcia-Debanc, C. (2008). Un apprentissage du lexique verbal par proximité sémantique : quand la représentation lexicale facilite la tâche d'écriture. In : F. Grossmann \& S. Plane (éds), Les apprentissages lexicaux. Lexique et production verbale. Lille : Presses Universitaires du Septentrion, 17-41.

Gangneux, M. \& Garcia-Debanc, C. (2013). Enseigner le lexique au cycle 3 : l'exemple des verbes de déplacement ( 2 dvd vidéo, $1 \mathrm{~cd})$. Toulouse : IUFM Midi-Pyrénées.

Garcia-Debanc, C. (2013). Les aides lexicales à la rédaction de textes : panorama historique et essai de typologie. In : C. Garcia-Debanc, C. Masseron \& C. Ronveaux (éds), Enseigner le lexique. Namur : Presses Universitaires de Namur, 273-300.

Garcia-Debanc, C. \& Aurnague, M. (2015). Effets d'un module d'enseignement des verbes de déplacement sur l'emploi de verbes spécifiques dans des productions écrites narratives à l'école primaire. Présentation au $83^{e}$ congrès de l'ACFAS, 23-25 mai 2015, Université du Québec à Rimouski.

Garcia-Debanc, C. \& Chourau, A. (2010). Enseigner les verbes de déplacement pour l'écriture de récits : de l'analyse de besoins à la mise en place d'activités en classe de CM2. Recherches, 53, 139-158.

Garcia-Debanc, C. \& Gangneux, M. (2015). L'Enseignement de la synonymie à l'école primaire. État des lieux et recherches innovantes pour une articulation entre enseignement du lexique et production écrite, Etudes de linguistique appliquée 178, 143-164.

Garcia-Debanc, C., Duvignau, K., Dutrait, C. \& Gangneux, M. (2009). Enseignement du lexique et production écrite. Un travail sur les verbes de déplacement à la fin de l'école primaire. Pratiques, 141-142, 208-232.

Grossmann, F. (2011). Didactique du lexique : état des lieux et nouvelles orientations. Pratiques, 149-150, 163-183.

Grossmann, F., Boch, F. \& Cavalla, C. (2008). Quand l'écriture n'empêche pas les sentiments...Quelques propositions pour mieux intégrer la dimension lexicale. In : F. Grossmann \& S. Plane (éds), Les apprentissages lexicaux. Lexique et production verbale. Lille : Presses Universitaires du Septentrion, 191-218.

Jackendoff, R. (1990). Semantic structures. Cambridge, MA : MIT Press.

Lakoff, G. (1987). Woman, fire and dangerous things: what categories reveal about the mind. Chicago : The University of Chicago Press.

Laparra, M. (2005). Capacités langagières en production non fictionnelle. Pratiques, 125/126, 139-156. 
Laur, D. (1991). Sémantique du déplacement et de la localisation en français : une étude des verbes, des prépositions et de leurs relations dans la phrase simple. Thèse de Doctorat, Université de Toulouse-Le Mirail.

Levin, B. (1993). English verb classes and alternations: a preliminary investigation. Chicago : The University of Chicago Press.

Levin, B. \& Rappaport, M. (1992). The lexical semantics of verbs of motion: the perspective from unaccusativity. In : I. M. Roca (éd), The thematic structure: its role in grammar. Berlin : Foris Publications, 247-269.

Levin, B. \& Rappaport, M. (1995). Unaccusativity: at the syntax-lexical semantics interface. Cambridge, MA : MIT Press.

Lieury, A. (1997). Mémoire et réussite scolaire. Paris : Dunod.

Masseron, C. (2001). Note critique sur les exercices de grammaire. Pratiques, 111/112, 209-236.

Masseron, C. (2008). Pour une topique de la peur: aspects psychologiques, sémiotiques, linguistiques. In : F. Grossmann \& S. Plane, Les apprentissages lexicaux. Lexique et production verbale. Lille: Presses Universitaires du Septentrion, 161-190.

Miller, G. \& Fellbaum, C. (1992). Semantic networks in English. In : B. Levin \& S. Pinker (éds), Lexical \& conceptual semantics. Oxford : Blackwell, 197-229.

Nonnon, E. (2012). La didactique du français et l'enseignement du vocabulaire dans vingt ans de revues de didactique du français langue première. Repères, 46, 33-72.

Randall, J.H. (2010). Linking: the geometry of argument structure. Dordrecht : Springer.

Rico Duarte, L., Syssau, A., Jiménez, M., Launay, M. \& Terrier, P. (2007). Problème d'accès ou déficit de stockage ? Apport du niveau de complexité de la tâche à l'étude des troubles sémantiques lors du vieillissement pathologique: le cas de la maladie d'Alzheimer. Canadian Journal on Aging/La Revue canadienne du vieillissement, 26 (3), 223-236.

Stosic, D. (2009). La notion de «manière » dans la sémantique de l'espace. Langages, 175, 103-121.

Talmy, L. (1985). Lexicalization patterns: semantic structure in lexical forms. In : T. Shopen (éd), Language typology and syntactic description (vol. 3) : grammatical categories and the lexicon. Cambridge : Cambridge University Press, 57-143.

Van der Linden, E. (2006) Lexique mental et apprentissage des mots. Revue française de linguistique appliquée, $1 / 2006$ (vol. XI), 33-44.

Viberg, Å. (2002). Basic verbs in second language acquisition. Revue Française de Linguistique Appliquée, VII, 2002/2, 51-69.

Wittgenstein, L. (1953). Philosophical investigations. New York : Mac Millan.

\footnotetext{
${ }^{1}$ Nous remercions les collègues de l'ESPE de l'Académie de Toulouse et les professeurs des écoles ayant pris part à la conception des activités évoquées dans cette étude et à leur mise en œuvre dans des classes. Ce travail a été réalisé dans le cadre d'une recherche collaborative Didacdép soutenue par l'IUFM Midi-Pyrénées. Notre reconnaissance va plus spécialement à Michel Gangneux, enseignant formateur à l'ESPE, qui a coordonné la captation vidéo de diverses séances dans les classes et l'édition du double dvd Enseigner le lexique au cycle 3 : l'exemple des verbes de déplacement qui en émane (Gangneux \& Garcia-Debanc, 2013) et à Lucile Jojou, étudiante de Master 2 Professeur des Ecoles, qui a effectué des transcriptions.

${ }^{2}$ La relation de troponymie - du grec tropos 'manière, mode' - lie un lexème verbal superordonné (ex : se déplacer) à un lexème sémantiquement apparenté quoique plus spécifique (ex : se traîner). Elle se substitue, dans le domaine des verbes, à la relation d'hypéronymie.

${ }^{3}$ Dans de nombreux cas, la classification des verbes des listes A et B a été précédée d'une activité individuelle de production de phrases comprenant ces lexèmes. Plusieurs séances de travail par groupes et de mise en commun des classifications ont été filmées (Gangneux \& Garcia-Debanc, 2013).

${ }^{4}$ Précisons, néanmoins, que deux verbes de la liste B (avancer, ramper) n’ont pas été fournis aux élèves par l'enseignante de CM1-CM2, qui a, par ailleurs, présenté ces verbes dans un ordre qui diffère substantiellement de
} 
celui initialement envisagé : patiner, glisser, se hisser, courir, dégringoler, se traîner, reculer, grimper, foncer, marcher, déraper, rouler, descendre, nager, skier, couler, dévaler, monter. Toutefois, cette modification ne présente, selon nous, pas de biais méthodologique particulier.

${ }^{5}$ Notons que les classes délimitées par les élèves ne comportent pas nécessairement d'en-tête ou label, surtout dans les productions individuelles analysées ici, ce qui n'enlève rien à leur cohérence interne éventuelle.

${ }^{6}$ Outre l'emploi de couler qui met en jeu une entité-cible malléable ou liquide, nous avons enregistré plusieurs usages de ce verbe se référant à une cible solide entraînée vers le bas d'un site au contenu malléable/liquide (ex. en CM1CM2 : Léon coule dans le sable mouvant; Léon coule dans la mer (...); Léon coule vers le fond de la piscine). C'est évidemment à ce deuxième usage que fait appel l'identification d'un médium aqueux. Les exemples ci-dessus et, plus généralement, ceux reproduits dans cet article apparaissent, la plupart du temps, avec une orthographe rétablie.

${ }^{7}$ Parmi les classifications « résiduelles », notons qu'un élève n'ayant sans doute pas compris la consigne, ou n'étant pas en mesure de l'appliquer, a retenu un critère non sémantique de catégorisation, celui de répartition des verbes en groupes morphologiques tel qu'utilisé traditionnellement en grammaire (verbes du $1^{\mathrm{er}}, 2^{\mathrm{e}}$ ou $3^{\mathrm{e}}$ groupe).

${ }^{8}$ Les cas d'hétérogénéité ou d'incohérence observés dans les classifications sont aussi imputables à un paramètre dont nous ne traiterons pas en détail dans ce travail, à savoir la propension des enfants à intégrer dans le sémantisme des verbes des éléments qui ne relèvent pas de celui-ci mais plutôt du contexte et de la pragmatique. Patiner et même reculer se verront ainsi associés au trait «vitesse ». Une partie des discussions suscitées par les mises en commun des classifications portent d'ailleurs sur cette difficulté (cf. section 5). En outre, précisons que les mécanismes d'élargissement des classes décrits dans la suite s'appliquent parfois aux en-têtes eux-mêmes et pas seulement aux verbes constitutifs d'une catégorie ou classe (le label en faisant exprès étend, par exemple, le concept d'opposition à une force au déplacement « contrôlé » d'une entité-cible animée).

${ }^{9}$ Autres exemples : Lou amorce la construction du regroupement intitulé en haut d'en bas avec les verbes monter (direction verticale supérieure) et (se) hisser (direction verticale supérieure + opposition à une force) pour ajouter ensuite les items (se) traîner (focalisation sur la seule opposition à une force) puis grimper (direction verticale supérieure + opposition à une force). Descendre (adjonction non motivée) apparaît aussi dans ce regroupement. Lino mobilise le même lien entre direction verticale supérieure et opposition à une force (voir plus loin) et associe, comme Lou, le verbe se traîner à des verbes dénotant un déplacement vers le haut (se hisser, grimper, monter).

${ }^{10}$ Le tiret unissant les propriétés mises en relation n'a pas valeur d'implication et les binômes pourraient tout aussi bien être présentés dans l'ordre inverse. Il s'agit, en quelque sorte, d'une relation symétrique.

${ }^{11}$ Autre exemple de consigne concernant le trait sémantique de direction : Léon se déplace(r)/bouge(r) dans une direction (glose) [Léon a/s'est avancé (vers la porte) (exemple)]; «Produis une phrase avec un (autre) verbe de déplacement indiquant la direction » (consigne à proprement parler).

${ }^{12}$ Des collocations telles que glisser en patinage ou patiner sur la glace recueillies, elles aussi, au cours de l'activité rituelle impliquant le trait sémantique de difficulté montrent que les verbes glisser et patiner dénotent, pour certains élèves, des procès dont la réalisation ne va pas de soi. Au-delà de la seule opposition à une force (cf. section 2), la difficulté est donc étendue ici au concept de déplacement contrôlé/non-contrôlé (voir également la note 8).

${ }^{13}$ Dans le cas de l'entraînement par une force, des prédications secondes ainsi que des propositions subordonnées ou coordonnées ont également été utilisées par les élèves pour essayer de traduire cette notion : Léon parvient à l'océan attiré par quelque chose; Léon monte au ciel, attiré par un aimant; Léon fuit car Lucile veut lui rendre le coup qu'il lui a donné (...); Léon est possédé et s'assoit sur le...

${ }^{14}$ Rappel de la hiérarchie obtenue dans le cadre de l'activité de classification des verbes (section 4.2) pour les quatre propriétés qui nous occupent : vitesse $(5)$ < difficulté/opposition à une force $(6)$ < direction verticale inférieure $(8)<$ direction verticale supérieure (9) < entrainement par une force (10). La hiérarchie des mêmes éléments de sens découlant du rituel «Léon » (Tableau 3, séances 2 et 3) est la suivante : direction $(40,90 \%)<$ difficulté/opposition à une force $(50 \%)$ < entraînement par une force $(54,16 \%)<$ vitesse $(82,60 \%)$. La direction pourrait, le cas échéant, être considérée comme l'élément dominant du premier ordonnancement pour peu que direction verticale inférieure et direction verticale supérieure soient fondues dans une même catégorie.

${ }^{15}$ La classification n'exige que le rapprochement et la comparaison de verbes préalablement fournis, sans autre contrainte que de construire des catégories. Le surplus de ressources attentionnelles et cognitives nécessaire à la réalisation du rituel tient sans doute au contenu de la consigne (compréhension du sens lexical glosé et de l'exemple éventuellement associé) et au transfert «analogique » entre cette consigne et l'exemple produit. Comme on peut le 
percevoir, les différences de complexité et de nature des processus cognitifs sous-tendant les deux types de tâches sont imputables aux tâches elles-mêmes et non à l'âge (elles pourraient, tout aussi bien, se manifester chez des sujets âgés : Rico Duarte et al., 2007). Nous remercions Liliana Rico Duarte pour ces précisions sur les corrélats cognitifs des activités soumises aux élèves. 